\title{
Prognostic significance of aberrant CD5 expression in B-cell leukemia
}

\author{
Kaveh Jaseb, ${ }^{1}$ Daryush Purrahman, ${ }^{1}$ Saeid Shahrabi, ${ }^{2}$ Majid Ghanavat, ${ }^{3}$ Hadi Rezaeean, ${ }^{1}$ \\ Najmaldin Saki ${ }^{1}$
}

${ }^{1}$ Thalassemia and Hemoglobinopathy Research Center, Research Institute of Health, Ahvaz Jundishapur University of Medical Sciences, Ahvaz, Iran; 'Department of Biochemistry and Hematology, Faculty of Medicine, Semnan University Of Medical Sciences, Semnan, Iran; ${ }^{3}$ Child Growth \& Development Research Center, Isfahan University of Medical Sciences, Isfahan, Iran

\begin{abstract}
Aberrant expression of CD5 (as a T-cell marker) is seen in some leukemia and lymphoma of B lineage origin. Given that the signaling resulting from the expression of this marker plays an essential role in the development of leukemia and lymphoma, evaluating the expression of this marker is of paramount importance. Therefore, our goal in this study was to investigate the prognostic importance of CD5 expression in B-cell leukemia and lymphoma. We evaluate CD5 expression in normal and leukemic B-cells by identifying relevant literature through a PubMed search (1998-2018) of English language papers using the terms: ' $C D 5$,' 'B-cell, 'Leukemia,' and 'Lymphoma.' We are doing this thorough comparison of results from CD5 positive and negative cases to make a correct decision about prognostic importance of CD5 expression in these malignancies. In a number of B-cell malignancies, CD5 is expressed in varying degrees. Due to the different origins and characteristics of these malignancies, the results of CD5 expression evaluations are heterogeneous and impossible to generalize. However, CD5 expression is sometimes associated with clinicopathologic findings, more invasive clinical course, and even resistance to treatment (specifically in DLBCL) among CD5-
\end{abstract}

Correspondence: Najmaldin Saki, Thalassemia and Hemoglobinopathy Research Center, Research Institute of Health, Ahvaz Jundishapur University of Medical Sciences, Ahvaz, Iran.

E-mail: najmaldinsaki@gmail.com

Key words: CD5; leukemia; lymphoma; prognosis.

Acknowledgments: we wish to thank all our colleagues in Shafa Hospital, Ahvaz Jundishapur University of Medical Sciences.

Contributions: NS conceived the manuscript and revised it; KJ, DP, SS, MG, HR wrote the manuscript and prepared the table.

Conflict of interest: the authors declare no conflict of interest.

Received for publication: 8 November 2018 .

Revision received: 26 December 2018.

Accepted for publication: 18 January 2019.

This work is licensed under a Creative Commons Attribution NonCommercial 4.0 License (CC BY-NC 4.0).

CCopyright K. Jaseb et al., 2019

Licensee PAGEPress, Italy

Oncology Reviews 2019; 13:400

doi:10.4081/oncol.2019.400 positive patients, which appears to be a function of CD5 signaling and its downstream factors such as STAT3. Depending on the type of malignancy, CD5 expression is associated with good or bad prognosis, which can be used as an auxiliary prognostic factor to assess the clinical course of B-cell malignancies. Moreover, the difference in expression levels of CD5 in a variety of B-cell malignancies allows for differential diagnosis of these malignancies, which can be helpful when diagnosis is difficult.

\section{Introduction}

CD5 (11q12.2) is a glycoprotein with a molecular weight of 67$\mathrm{kDa}$. Normally, CD5 is strongly expressed in all types of T lymphocytes but weakly expressed in a small subgroup of B lymphocytes called B-1a comprising $25 \%$ of B lymphocytes in adults. ${ }^{1-3}$ In B lymphocytes, CD5 marker is located in physical binding with B-cell receptor (BCR) complex, which is a negative regulator of downstream signaling pathways. ${ }^{4}$ In addition, by triggering signaling pathways such as Erk1/2 phosphorylation via activating mitogenactivated protein kinase (MAPK), signal transducer and activator of transcription 3 (STAT3), and nuclear factor of activated $\mathrm{T}$ cells (NFAT), CD5 is directly involved in the induced production of Interleukine-10 (IL-10), which is known as an anti-apoptotic cytokine causing autocrine protection against autoimmune diseases. ${ }^{5-7}$ CD5 seems to have a potential association with the effects of STAT3 expression (especially malignancy) given the key roles (such as growth, survival, tumorigenicity, and transformation) of STAT3 in the cell and the relationship of its feed-forward loop with CD5. ${ }^{8}$ In the meantime, in some B lymphocyte malignancies, the aberrant expression of CD5 (as T-cell marker) has been reported, including chronic lymphocytic leukemia (CLL) and Mantle Cell Lymphoma (MCL) in which this condition is universally observed. However, in rare cases of B-Prolymphocytic Leukemia (B-PLL), Diffuse Large B-cell Lymphoma (DLBCL), Follicular Lymphoma (FL), Splenic marginal zone lymphoma (SMZL), and even B-Acute Lymphoblastic Leukemia (B-ALL), the aberrant CD5 expression is also reported. ${ }^{9}$ Although these malignant CD5 positive B-cells have different origins, it is clear that the aberrant expression of CD5 plays certain roles that can affect the clinical course of the disease. ${ }^{6}$ Therefore, in this review article, we examine the expression of CD5 and its possible role in B lymphocyte malignancy with a prognostic approach (Figure 1).

\section{B-chronic lymphocytic leukemia}

CLL is a type of CD5-positive B lymphocytes malignancy, which is diagnosed when there is an accumulation of malignant 
cells in peripheral blood (PB), bone marrow (BM), and lymph nodes. ${ }^{10}$ Reduction of apoptosis and increase in proliferation lead to cellular accumulation. ${ }^{11}$ But what is the role of CD5 in this malignancy? CD5 can be involved in the development and progression of this malignancy in two potential ways: the defects in negative regulation of BCR-induced signaling confirmed by PerezChacon et al. and the signaling pathways triggered by CD5 marker itself.,12,13 Phosphorylation and activation of STAT3 via B-cell linker protein (BLNK) and CK2 complex is a well-known signaling pathway. Following the phosphorylation of STAT3, this factor goes into the nucleus and turns on the target genes. ${ }^{10,14}$ The expression of IL-10 gene, which is significantly associated with CLL progression, is induced in this way and acts as a growth factor for CLL cells contributing to deteriorating clinical course of the disease and patients' autoimmunity (which occurs in 10\% of CLL cases) by increasing proliferation and protection against apoptosis. ${ }^{14-16}$ However, in a study by Gary-gouy et al., new indications of CD5 function in CLL showed that CD5 has a regulatory effect on the expression of several genes in CLL cells, which is linked to the involvement of malignant cells in different sites. For example, they found that the genes involved in cell migration (such as CCR7) as well as those involved in homing (such as SDF-1) are respectively upregulated and downregulated under the influence of CD5. Since the interaction of CCR7 with its ligand (i.e. integrin) contributes to the involvement of lymph nodes in these patients, ${ }^{15}$ it can be concluded that CD5 plays an indirect role in the involvement of patients' lymph nodes. It is worth noting that CD5 expression in CLL is not constant and that the expression level of this marker can be reduced in presence of pharmacologic agents such as rosiglitazone and atorvastatin as well as physiological ones such as IL-4 secreted by $\mathrm{T}$ lymphocytes. It has been found that IL-4 does this through direct inhibition of CD5 mRNA, ${ }^{17,18}$ but there is no clear understanding of the mechanism of action of the mentioned drugs. However, they are assumed to suppress CD5 by changing the cytokine network. In addition, reports show that CD5 is not expressed in about $10 \%$ of CLL cases, ${ }^{19}$ but the findings are not consistent. For example, a lower rate of lymph node involvement along with splenomegaly and hemolytic anemia was noted in CD5negative cases, but a more recent study showed that the outcome of CD5 negative and positive patients was not different. ${ }^{19,20}$ That is why the precise prognosis of CD5 expression in CLL is not clear yet. However, due to the direct effect of CD5 on IL-10 expression and the pivotal role of this cytokine in CLL, it would seem that any factor that can inhibit CD5 or its dependent agents (such as CD5 or IL-10 mRNA regulation), or the induction of a decoy receptor for IL-10 can help improve the clinical course of these patients.

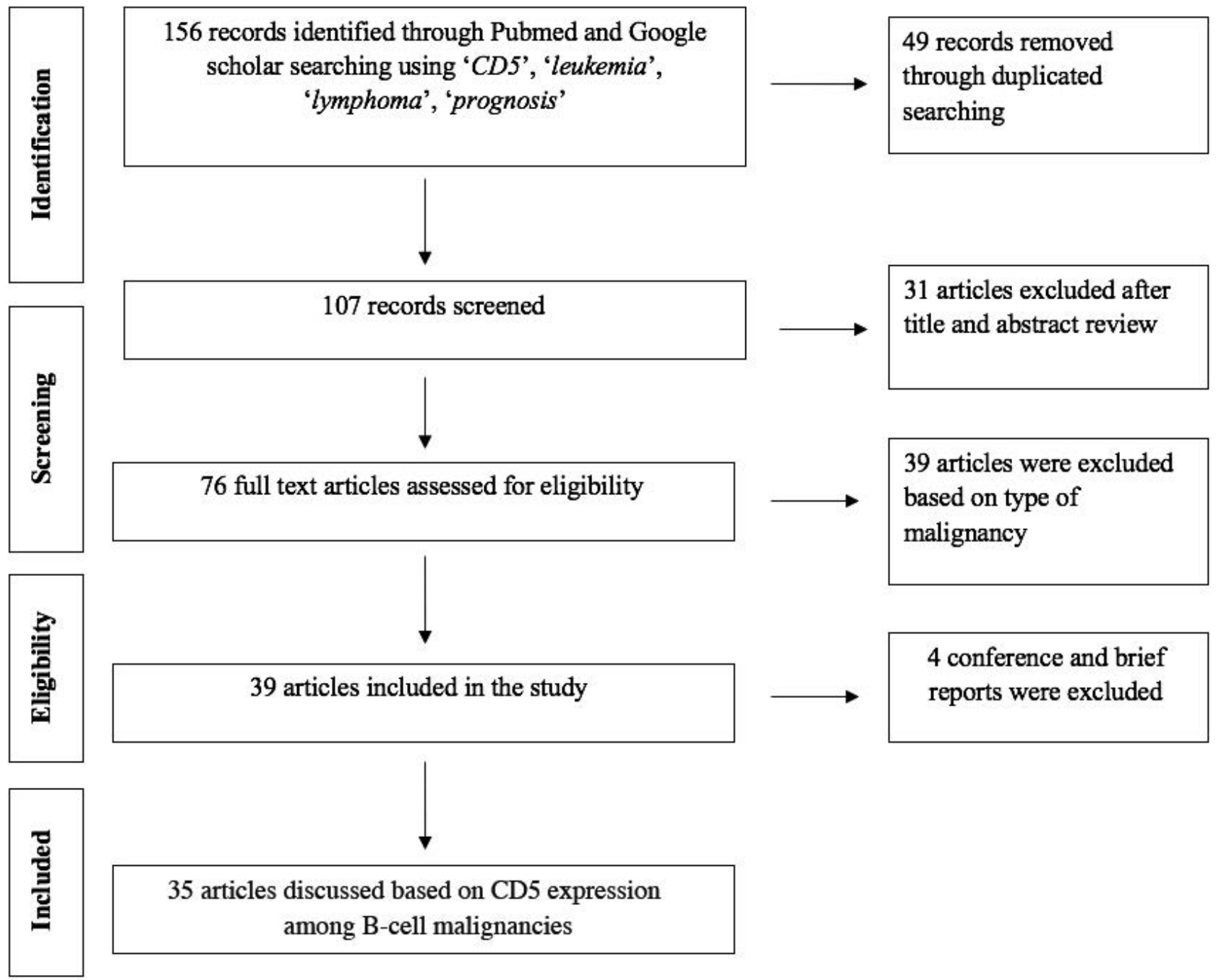

Figure 1. Flow chart of study selection and inclusion process. 


\section{B-pro lymphocytic leukemia}

B Pro Lymphocytic leukemia (Pro-lymphocyte $>55 \%$ ) is associated with manifestations such as huge splenomegaly and lymphadenopathy and is observed in both PLLs resulting from CLL transformation and de novo PLLs (with $>50 \%$ incidence). ${ }^{21}$ The PLLs arising from CLL remain CD5-positive, but de novo PLLs are usually CD5 negative. Due to limited number of reports, it is not possible to make precise statements regarding the prognosis of CD5 expression in this malignancy. However, in a study involving CD5-positive and CD5-negative PLLs, it was found that there are $t(11 ; 14)$ cytogenetic abnormalities in most (4 out of 5 ) cases of CD5-positive PLL, which are characteristic of MCL. This finding implies two important points. First, the expression of CD5 in PLL may be associated with cytogenetic abnormalities. Second, when observing $\mathrm{t}(11 ; 14)$, it is necessary to be careful with CD5-positive PLL cases to avoid misdiagnosis. ${ }^{22,23}$

\section{Mantle cell lymphoma}

MCL makes up $10 \%$ of non-Hodgkin lymphoma (NHL) types and its diagnostic biomarker is the presence of $t(11 ; 14)$, which is observed as an overexpression of Cyclin D1. ${ }^{24}$ This lymphoma has several types such as Classical (the most common variant), Small, Marginal-like, Pleomorphic, and Blastoid, which are always CD5positive due to originating from Mantle Zone cells of lymphoid follicles. However, some reports indicate that CD5 expression is sometimes negative in the latter two cases (i.e., Pleomorphic and Blastoid). Given the frequently high genetic complexity of Blastoid and Pleomorphic variants, these cytogenetic abnormalities seem to be the cause of the loss of CD5 expression in these variants. A point to note is that the results of studies in CD5-negative blastoid variants indicate an invasive clinical course of these patients, the cause of which is still unclear. ${ }^{25-27}$ On the other hand, the findings of Todorovic et al. show that MCL patients with CD5 levels less than $80 \%$ have a shorter overall survival than those with more than $80 \%{ }^{28}$ According to these results, the expression of CD5 in MCL seems to have a more favorable prognosis as opposed to other non-Hodgkin lymphomas. In other words, it may be possible to consider the loss of CD5 expression as an indication of the invasiveness of MCL's clinical course.

\section{Diffuse large B-cell lymphoma}

DLBCL is the most common type of NHL that is divided into two subgroups in terms of CD5 expression, ${ }^{2}$ namely the classic subgroup (involving most patients) with no CD5 expression, and the other subgroup with CD5 expression that includes 5-10\% of DLBCL patients. ${ }^{29}$ Of course, due to morphological transformation of CD5-positive CLL, DLBCLs are of two types: de novo $\mathrm{CD}^{+}$DLBCL and Richter syndrome. ${ }^{30}$ There are well-known differences between these two types, which allow their differentiation. For example, the rearrangement of BCL-6 is in favor of de novo $\mathrm{CD}^{+}$DLBCL detection. ${ }^{30}$ However, in the present review, we only deal with CD5-positive DLBCL regardless of its origin, and the results show unfavorable clinicopathologic features of this type compared to classical type. For example, central nervous system (CNS) relapse along with extra-nodal position (e.g., bone marrow) is more frequent in patients with CD5-positive DLBCL. 2,7,31 Analysis of comparative genomic hybridization shows the difference in the pattern of gain and loss of chromosomes in CD5-posi- tive DLBCL patients with the classic type. For example, the loss of P16INK4a (9q21) makes the progression of DLBCL more pronounced in this type. ${ }^{32}$ These findings along with the lack of a significant relationship between CD5 and other biomarkers involved in prediction of DLBCL outcome (such as MYC Rearrangement) suggest the poor and independent prognostic role of CD5 marker in DLBCL. 2,32 The poor prognosis can be attributed to the negative regulatory role of this marker in BCR-induced signaling. The overexpression of BCL2, STAT3, and NF-KB (due to excessive BCR stimulation) in CD5-positive DLBCL cases confirms this hypothesis. ${ }^{2,7}$ Also, it is worth noting that unlike patients with classic type, CD5-positive DLBCL patients do not respond adequately to the treatment by Rituximab regimen. ${ }^{32}$ Since Rituximab targets the CD20 marker on malignant cell surface, it is believed that downstream CD5 signaling leads to a reduction in the expression of CD20 and consequently to a reduction in the effect of drug. Therefore, the use of CD5 inhibitors would probably result in a favorable response to Rituximab in these patients.

\section{Follicular lymphoma}

FL is one of the most common types of NHL. Using a set of diagnostic panels including immunophenotypes (positivity of Bcell markers and negative CD5 and CD23), histology, and cytogenetics [the presence of $\mathrm{t}(14 ; 18)$ in $>80 \%$ of cases], FL is differentiated from other lymphoproliferative disorders of B lymphocytes. ${ }^{33}$ However, in recent years, due to the increased sensitivity and specificity of monoclonal antibodies, CD5-positive FL cases have been rarely reported. MCL and CLL should be ruled out to diagnose CD5-positive FL, while the expression of CD10 and the lack of expression of Cyclin D1, CD43, and IgD are in favor of FL diagnosis. ${ }^{34}$ The reason for the expression of CD5 in this malignancy is still unknown, but it may be due to genetic disorders such as gain of chromosomes 1,3,5,7,10, X, and 18q, and loss of chromosomes $6 \mathrm{p}, \mathrm{y}$, and 13 , which are widely observed in cytogenetics of this type. ${ }^{35}$ It should be noted that due to the rarity of CD5-positive FL cases, there is no accurate understanding of its pathogenesis. However, limited studies have shown the difference in clinicopathologic characteristics of these two types. For example, the involvement of several extra-nodal sites such as neck and gastrointestinal tract tends to be higher in CD5-positive patients. Also, CD5-positive FL patients have a more advanced clinical course with a higher International Prognostic Index (IPI), a shorter progression-free survival (PFS), and a lower frequency of $\mathrm{t}(14 ; 18)$ as opposed to the classic FL. ${ }^{36,37}$ Nevertheless, what needs to be considered here is the higher involvement of the peripheral blood (PB), which has been mentioned in various studies. ${ }^{35,37}$ To explain this situation, it appears that CD5 elevates the mobilization of malignant cells by altering their interaction with an extracellular matrix as well as downregulating some cell adhesion genes, such as integrin. Perhaps the evaluation of expression levels of markers involved in cellular attachments such as CD44 in patients with CD5-positive FL can help clarify this issue.

\section{Splenic marginal zone lymphoma}

SMZL is a rare and indolent lymphoma classified by World Health Organization (WHO) as a subgroup of marginal zone lymphoma and includes about $1 \%$ of NHL cases. ${ }^{38}$ SMZL is characterized by the presence of splenomegaly as well as BM and PB involvement in most patients. Malignant cells in SMZL express 
class B markers (CD19, CD20, CD22, and CD79a), but are negative for CD10, CD23, CD5, CD43 and Cyclin D1. However, recent reports indicate that about $20 \%$ of SMZL patients express CD5. ${ }^{39}$ Due to the low frequency of this malignancy, limited studies have been done on it, and interestingly, their results are not consistent and sometimes even contradictory. For example, earlier studies referred to the poor prognosis of CD5 expression and associated it with an invasive clinical course. In this regard, a case report mentioned four deaths, of which three had CD5-positive SMZL. ${ }^{39,40}$ However, more recent studies address the lack of distinction of clinicopathologic features such as survival and outcome between CD5-positive and the classic type ${ }^{41-43}$ In addition, findings related to cytogenetic pattern remain controversial in different studies, with the results of some researches indicating differences in the cytogenetic pattern; for example, Del7q and Del17p13 are unique to the classic and CD5-positive SMZLs. ${ }^{39,41}$ However, the results of studies by Baseggio et al. suggest the similarity in the karyotype specification since they observed anomalies such as Del7q as well as trisomy 3 and 18 in both types. ${ }^{43}$ According to these results, CD5 expression prognosis in SMZL is not clear and calls for further studies in the future.

\section{B-acute lymphoblastic leukemia}

B-ALL is a malignancy of hematopoietic precursor cells committed to B-linage, but rarely are $\mathrm{T}$ and $\mathrm{NK}$ antigens present in immunophenotypes of these leukemia cells. ${ }^{11}$ In a case report, two CD5-positive B-ALL patients had a poor outcome with cytogenetic abnormalities of Trisomy 22 (in one case). ${ }^{44}$ Another study looked at the frequency and outcome of the expression of T-cell antigens, including CD5, in B-ALL cases. It was found that six out of 134 cases had CD5 expressions. To evaluate the outcome of aberrant expression of T antigens in B-ALL patients, a Cox regression multivariate analysis was performed indicating a higher level of relapse risk in CD5-expressing subjects as opposed to those expressing other T-cell antigens. ${ }^{45,46}$ According to these results, CD5 can be considered a poor prognostic factor for B-ALL, which seems to be related to cytogenetic abnormalities (Table 1). ${ }^{47,48}$

\section{Discussion and future perspectives}

CD5 is expressed in some B leukemia/lymphoma types, which is thought to play an important role in the fate of malignant cells and thus in the clinical course due to its internal cellular signaling capabilities. In our review, we revealed that the aberrant expression of CD5 in B leukemia/lymphoma yields a good prognosis (in MCL) and a bad one (in DLBCL, FL, B-ALL and possibly B-CLL) depending on the type of malignancy, indicating the prognostic importance of its expression in monitoring of B-cell leukemia. $7,22,24,46$ In poor prognoses, with a more aggressive clinical course, the resistance to chemotherapy (specifically in DLBCL) and increased rate of malignant cell involvement are linked to various sites such as lymph nodes, which can be due to CD5 function in regulating essential cell markers, including markers involved in migration, such as integrin- $\beta 1$ and CCR7. On the other hand, the expression of CD5 in MCL has a better prognosis compared to lack of its expression, the exact cause of which is still unknown. $2,7,15,24,35$ It is worth noting that differential diagnosis is necessary due to different natures of these malignancies, which include a spectrum of malignancies from indolent (like FL) to aggressive (such as MCL). However, atypical cases can challenge differential diagnosis and increase the risk of misdiagnosis. In these cases, the use of quantitative CD5 assessment with a sensitive flow cytometry method can be helpful in the differential panel specifically designed for the differentiation of FL from MCL since studies show a significant difference in the level of CD5 expression between FL and MCL with CLL (less than $10^{2}$ in FL and about $\leqq 10^{4}$ in CLL and MCL according to CD5 antibody per cell value). ${ }^{27,47}$

\section{Conclusions}

Aberrant expression of CD5 in B leukemia/lymphoma is associated with good or bad prognosis depending on the type of malignancy, which can be used either in diagnosis or during monitoring for the prognosis of malignancy and also in the differential panel.

Table 1. An overview of aberrant CD5 expression in B-cell malignancies.

\begin{tabular}{|c|c|c|c|c|c|}
\hline Malignancy & Genetic abnormalities & $\%$ & Pathogenesis & Prognosis & Ref. \\
\hline$B-C L L$ & Not mentioned & $>90 \%$ & $\begin{array}{l}\text { Induces IL-10 production } \\
\text { and defect in negative } \\
\text { regulation of BCR }\end{array}$ & CR & 6,13 \\
\hline B-PLL & $\mathrm{T}(11 ; 14)$ & $\begin{array}{l}\text { One-third of } \\
\text { Cases }\end{array}$ & NR & CR & 22 \\
\hline$M C L$ & $\begin{array}{l}\text { Great genetic complexity in Blastoid } \\
\& \text { Pleomorphic variants }\end{array}$ & Most cases & NR & Good & $4,26-28$ \\
\hline$D L B C L$ & Gain and loss of chromosome & $5-10 \%$ & $\begin{array}{l}\text { Unfavorable Clinicopathologic } \\
\text { features }\end{array}$ & Poor & $2,7,31,32$ \\
\hline$F L$ & $\begin{array}{l}\text { Gain in CHr. 1, 3, 5, X and loss } \\
\text { of Chr. 2, 13, Y chromosome }\end{array}$ & Rare & $\begin{array}{l}\text { Different Clinicopathologic } \\
\text { features from classic type }\end{array}$ & Poor & $33-36,47$ \\
\hline SMZL & Del7q, Del17p13 & $20 \%$ & NR & CR & $38-43$ \\
\hline$B-A L L$ & Trisomy 22 & Rare & NR & Poor & $45,46,48$ \\
\hline
\end{tabular}

B-CLL, B-chronic lymphocytic leukemia; B-PLL, B-prolymphocytic leukemia; MCL, mantle cell lymphoma; DLBCL, diffuse large b-cell lymphoma; FL, follicular lymphoma; SMZL, splenic marginal zone lymphoma; B-ALL, b-acute lymphoblastic leukemia; NHL, non-Hodgkin lymphoma; BCR, B-cell receptor; Chr, chromosome; IL-10, interleukin-10; CR, controversial reported; NR, not reported. 


\section{References}

1. Dalloul A. CD5: a safeguard against autoimmunity and a shield for cancer cells. Autoimmun Rev 2009;8:349-53.

2. Thakral B, Medeiros LJ, Desai P, et al. Prognostic impact of CD5 expression in diffuse large B-cell lymphoma in patients treated with rituximab-EPOCH. Eur J Haematol 2017;98:41521.

3. Consuegra-Fernández M, Aranda F, Simões I, et al. CD5 as a target for immune-based therapies. Crit Rev Immunol 2015;2:35.

4. Zhang C, Xin H, Zhang W, et al. CD5 binds to interleukin-6 and induces a feed-forward loop with the transcription factor STAT3 in B cells to promote cancer. Immun 2016;44:913-23.

5. Mageed RA, Garaud S, Taher TE, et al. CD5 expression promotes multiple intracellular signaling pathways in B lymphocyte. Autoimmun Rev 2012;11:795-8.

6. Garaud S, Taher TE, Debant M, et al. CD5 expression promotes IL-10 production through activation of the MAPK/Erk pathway and upregulation of TRPC1 channels in B lymphocytes. Cell Mol Immunol 2016 [Epub ahead of print].

7. Xu-Monette ZY, Tu M, Jabbar KJ, et al. Clinical and biological significance of de novo CD5+ diffuse large B-cell lymphoma in Western countries. Oncotarget 2015;6:5615.

8. Rozovski U, Harris DM, Li P, et al. Constitutive phosphorylation of STAT3 by the CK2-BLNK-CD5. Complex 2017;15:610-8.

9. Mutreja D, Pati HP, Bansal D, et al. Aberrant immunophenotypic expression of CD5 in a case of B acute lymphoblastic leukemia: a case report. Indian J Hematol Blood Transf 2014;30:212-4.

10. Perez-Chacon G, Vargas JA, Jorda J, et al. CD5 provides viability signals to B cells from a subset of B-CLL patients by a mechanism that involves PKC 2007;31:183-93.

11. Tibaldi E, Brunati A, Zonta F, et al. Lyn-mediated SHP-1 recruitment to $\mathrm{CD} 5$ contributes to resistance to apoptosis of $\mathrm{B}$ cell. Chron Lymphocyt Leuk Cells 2011;25:1768.

12. Alhakeem SS, Sindhava VJ, McKenna MK, et al. Role of B cell receptor signaling in IL-10 production by normal and malignant B-1 cells. 2015;1362:239-49.

13. Perez-Chacon G, Vargas JA, Jorda J, et al. CD5 does not regulate the signaling triggered through BCR in B cells from a subset of B-CLL patients. Leuk Lymph 2007;48:147-57.

14. Garaud S, Morva A, Lemoine S, et al. CD5 promotes IL-10 production in chronic lymphocytic leukemia B cells through STAT3 and NFAT2 activation. J Immunol 2011;186:4835-44.

15. Gary-Gouy H, Sainz-Perez A, Marteau J-B, et al. Natural phosphorylation of CD5 in chronic lymphocytic leukemia B cells and analysis of CD5-regulated genes in a B cell line suggest a role for $\mathrm{CD} 5$ in malignant phenotype. J Immunol 2007; 179:4335-44.

16. Laurenti L, Vannata B, Innocenti I, et al. The use of monoclonal antibodies in the treatment of autoimmune complications of chronic lymphocytic leukemia. Med J Hematol Infect Dis 2013;5:1.

17. Murphy M, Hoss D. Cutaneous involvement by CD5-negative chronic lymphocytic leukemia (CLL)-aberrant phenotype or false negativity? Implications for immunohistochemical testing on paraffin-embedded skin specimens. J Cutan Pathol 2011;38:73-5.

18. Yavasoglu I, Sargin G, Kadikoylu G, et al. The activity of atorvastatin and rosiglitazone on CD38, ZAP70 and apoptosis in lymphocytes of B-cell chronic lymphocytic leukemia in vitro. Med Oncol 2013;30:603.
19. Efstathiou S, Tsioulos D, Zacharos I, et al. The prognostic role of CD5 negativity in B-cell chronic lymphocytic leukaemia: a case-control study. Leukemia 2002;32:209-18.

20. Romano C, Sellitto A, Chiurazzi F, et al. Clinical and phenotypic features of CD5-negative B cell chronic lymphoproliferative disease resembling chronic lymphocytic leukemia. Leukemia 2015;101:67-74.

21. Hercher C, Robain M, Davi F, et al. A multicentric study of 41 cases of B-prolymphocytic leukemia: two evolutive forms: the Groupe Français d'Hématologie Cellulaire. Leukemia 2001;42:981-7.

22. Kar R, Kumar R, Tyagi SJTJoH. De-novo CD5+ B-prolymphocyte leukemia (PLL) presenting at younger age with favourable outcome. Leuk Lymph 2008;25:3.

23. Ruchlemer R, Parry-Jones N, Brito-Babapulle V, Attolico I, Wotherspoon AC, Matutes E, et al. B-prolymphocytic leukaemia with $\mathrm{t}(11 ; 14)$ revisited: a splenomegalic form of mantle cell lymphoma evolving with leukaemia. 2004; 125:330-6.

24. Wohlschlaeger C, Lange K, Merz H, Feller A. Aberrant immunophenotypes of mantle cell lymphomas. Leuk Lymph 2003;44:269-73.

25. Elyamany G, Alzahrani AM, Al Mussaed E, et al. De Novo CD5 Negative Blastic Mantle Cell Lymphoma Presented with Massive Bone Marrow Necrosis without Adenopathy or Organomegaly. 2015;2015.

26. Miao Y, Lin P, Wang W, et al. CCND1-IGH FusionAmplification and MYC Copy Number Gain in a Case of Pleomorphic Variant Mantle Cell Lymphoma. Am J Clin Pathol 2016;146:747-52.

27. Yamada T, Goto N, Tsurumi H, et al. Mantle cell lymphoma with a unique pattern of CD5 expression: a case report with review of the literatures. Med Mol Morphol 2014;47:169-75.

28. Todorovic M, Balint B, Andjelic B, et al. Outcome prediction of advanced mantle cell lymphoma by international prognostic index versus different mantle cell lymphoma indexes: one institution study. Med Oncol 2012;29:2212-9.

29. Chuang W-Y, Chang H, Shih L-Y, Wang P-N, Chang Y-S, Lin $\mathrm{T}-\mathrm{L}$, et al. CD5 positivity is an independent adverse prognostic factor in elderly patients with diffuse large B cell lymphoma. Virchows Archiv 2015;467:571-82.

30. Taniguchi M, Oka K, Hiasa A, et al. De novo CD5+ diffuse large B-cell lymphomas express VH genes with somatic mutation. Blood 1998;91:1145-51.

31. Zhang X, Sun M, Zhang L, Shao H. Primary hepatosplenic CD5-positive diffuse large B-cell lymphoma: a case report with literature review. Int J Clin Exper Pathol 2013;6:985.

32. Ennishi D, Takeuchi K, Yokoyama M, et al. CD5 expression is potentially predictive of poor outcome among biomarkers in patients with diffuse large B-cell lymphoma receiving rituximab plus CHOP therapy. Ann Oncol 2008;19:1921-6.

33. Mayson E, Saverimuttu J, Cartwright K. CD5-positive follicular lymphoma: prognostic significance of this aberrant marker? Intern Med J 2014;44:417-22.

34. Barry TS, Jaffe ES, Kingma DW, et al. CD5+ follicular lymphoma: a clinicopathologic study of three cases. Am J Clin Pathol 2002;118:589-98.

35. Li Y, Hu S, Zuo Z, et al. CD5-positive follicular lymphoma: clinicopathologic correlations and outcome in 88 cases. Modern Pathology 2015;28:787-98.

36. Sonoki T, Ishihara S, Uneda S, et al. Aggressive CD5-positive B-cell lymphoma after remission of CD5-negative follicular lymphoma with distinct immunoglobulin heavy chain rearrangement and translocation. Int J Hematol 2008;88:299- 
303.

37. Miyoshi H, Sato K, Yoshida M, et al. CD5-positive follicular lymphoma characterized by CD25, MUM1, low frequency of $t$ $(14 ; 18)$ and poor prognosis. Pathol Int 2014;64:95-103.

38. Raya J, Ruano J, Bosch J, et al. Splenic marginal zone lymphoma-a clinicopathological study in a series of 16 patients. Hematology 2008;13:276-81.

39. Gimeno E, Salido M, Solé F, et al. CD5 negative and CD5 positive splenic marginal B-cell lymphomas have differential cytogenetic patterns. Leuk Res 2005;29:98.

40. Giannouli S, Paterakis G, Ziakas PD, et al. Splenic marginal zone lymphomas with peripheral CD5 expression. Haematologica 2004;89:113-4.

41. Salido M, Baró C, Oscier D, et al. Cytogenetic aberrations and their prognostic value in a series of 330 splenic marginal zone B-cell lymphomas: a multicenter study of the Splenic B-Cell Lymphoma Group. Blood 2010;2:267476.

42. Kojima M, Sato E, Oshimi K, et al. Characteristics of CD5positive splenic marginal zone lymphoma with leukemic manifestation; clinical, flow cytometry, and histopathological findings of 11 cases. J Clin Exp Hematopathol 2010;50:107-12.
43. Baseggio L, Traverse-Glehen A, Petinataud F, et al. CD5 expression identifies a subset of splenic marginal zone lymphomas with higher lymphocytosis: a clinico-pathological, cytogenetic and molecular study of 24 cases. Haematologica 2010;95:604-12.

44. Peterson MR, Noskoviak KJ, Newbury R. CD5-positive B-cell acute lymphoblastic leukemia. Pediatr Develop Pathol 2007;10:41-5.

45. Hussein S, Gill KZ, Sireci AN, et al. Aberrant T-cell antigen expression in B lymphoblastic leukaemia. Br J Haematol 2011;155:449-56.

46. Ahmed D, Ahmed TA, Ahmed S, et al. CD5-positive acute lymphoblastic leukemia. J Coll Physicians Surg Pak 2008;18:310-1.

47. Challagundla P, Jorgensen JL, Kanagal-Shamanna R, et al. Utility of quantitative flow cytometry immunophenotypic analysis of CD5 expression in small B-cell neoplasms. Archiv Pathol Lab Med 2014;138:903-9.

48. Huang PY-H, Kohnke P, Belov L, et al. Profiles of surface mosaics on chronic lymphocytic leukemias distinguish stable and progressive subtypes. J Pharm Pharm Sci 2013;16:231-7. 